Received: 29 September 2017

Accepted: 11 January 2018

Published online: 25 January 2018

\title{
Isolation and characterization of novel mutations in the pSC101 origin that increase copy number
}

Mitchell G. Thompson ${ }^{1,2,3}$, Nima Sedaghatian ${ }^{1,2}$, Jesus F. Barajas ${ }^{2,4}$, Maren Wehrs ${ }^{1,2}$, Constance B. Bailey ${ }^{1,2}$, Nurgul Kaplan ${ }^{1,2,4}$, Nathan J. Hillson ${ }^{1,2,4}$, Aindrila Mukhopadhyay ${ }^{1,2}$ \& Jay D. Keasling ${ }^{1,2,5,6,7}$

pSC101 is a narrow host range, low-copy plasmid commonly used for genetically manipulating Escherichia coli. As a byproduct of a genetic screen for a more sensitive lactam biosensor, we identified multiple novel mutations that increase the copy number of plasmids with the pSC101 origin. All mutations identified in this study occurred on plasmids which also contained at least one mutation localized to the RepA protein encoded within the origin. Homology modelling predicts that many of these mutations occur within the dimerization interface of RepA. Mutant RepA resulted in plasmid copy numbers between $\sim 31$ and $\sim 113$ copies/cell, relative to $\sim 5$ copies/cell in wild-type pSC101 plasmids. Combining the mutations that were predicted to disrupt multiple contacts on the dimerization interface resulted in copy numbers of $\sim 500$ copies/cell, while also attenuating growth in host strains. Fluorescent protein production expressed from an arabinose-inducible promoter on mutant origin derived plasmids did correlate with copy number. Plasmids harboring RepA with one of two mutations, E83K and N99D, resulted in fluorescent protein production similar to that from p15a- ( 20 copies/cell) and ColE1- ( 31 copies/cell) based plasmids, respectively. The mutant copy number variants retained compatibility with p15a, pBBR, and ColE1 origins of replication. These pSC101 variants may be useful in future metabolic engineering efforts that require medium or high-copy vectors compatible with $\mathrm{p} 15 \mathrm{a}$ - and ColE1-based plasmids.

Metabolic engineering of bacterial hosts relies heavily on the heterologous expression of proteins from plasmids that replicate separately from the host chromosome. In order to achieve pathway balance, researchers use a variety of genetic control systems, including variable origins of replication, to control expression level ${ }^{1}$. Changing the origin of replication of a plasmid provides a convenient means of controlling the magnitude of expression of a heterologous pathway, and can dramatically change the performance of the engineered system within a host ${ }^{2,3}$. Plasmids with compatible origins of replication allow metabolic engineers to divide pathways into multiple parts able to be maintained at different copy numbers, thereby allowing for extra flexibility when trying to ensure maximum flux through a pathway ${ }^{3}$. Having a library of well characterized, orthogonal origins with different copy numbers therefore enables engineers to rapidly alter the characteristics of pathways in pursuit of achieving high product titers ${ }^{1}$.

The pSC101 origin is a low-copy, narrow host-range origin that is often used in synthetic biology and metabolic engineering ${ }^{1}$. Low-copy vectors offer many advantages for metabolic engineering, such as low metabolic burden on the host, tight control of gene expression, and segregational stability ${ }^{4}$. The pSC101 origin regulates its

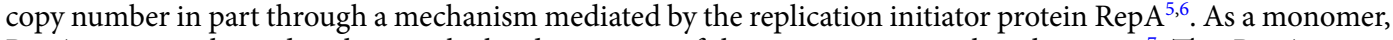
RepA initiates plasmid replication by binding to one of the many iterons within the origin ${ }^{7}$. This RepA-iteron

${ }^{1}$ DOE Joint BioEnergy Institute, 5885 Hollis Street, Emeryville, CA, 94608, USA. ${ }^{2}$ Biological Systems \& Engineering Division, Lawrence Berkeley National Laboratory, Berkeley, CA, 94720, USA. ${ }^{3}$ Department of Plant and Microbial Biology, University of California, Berkeley, CA, 94720, USA. ${ }^{4}$ DOE Agile BioFoundry, Emeryville, CA, 94608, USA. ${ }^{5}$ Department of Bioengineering, University of California, Berkeley, CA, 94720, USA. ${ }^{6}$ Department of Chemical and Biomolecular Engineering, University of California, Berkeley, CA, 94720, USA. ${ }^{7}$ The Novo Nordisk Foundation Center for Biosustainability, Technical University of Denmark, Denmark, Building 220 Kemitorvet, 2800Kgs Lyngby, Denmark. Mitchell G. Thompson and Nima Sedaghatian contributed equally to this work. Correspondence and requests for materials should be addressed to J.D.K. (email: jdkeasling@lbl.gov) 
A

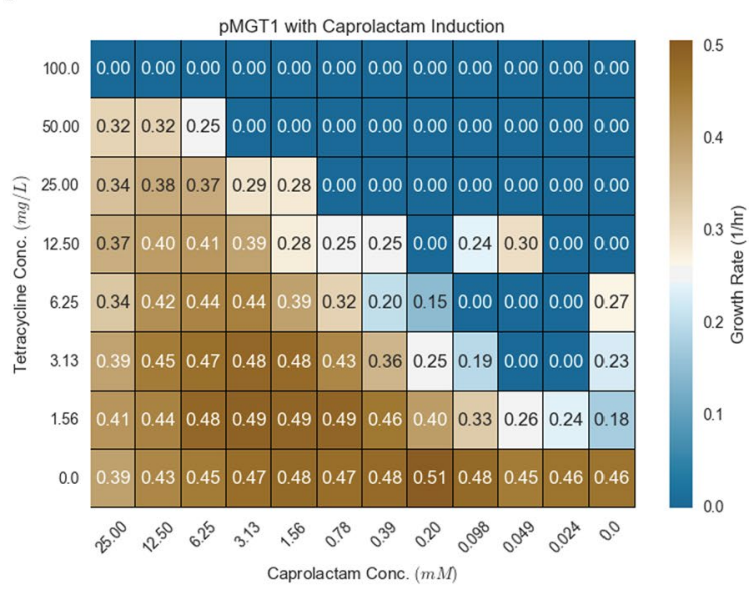

B

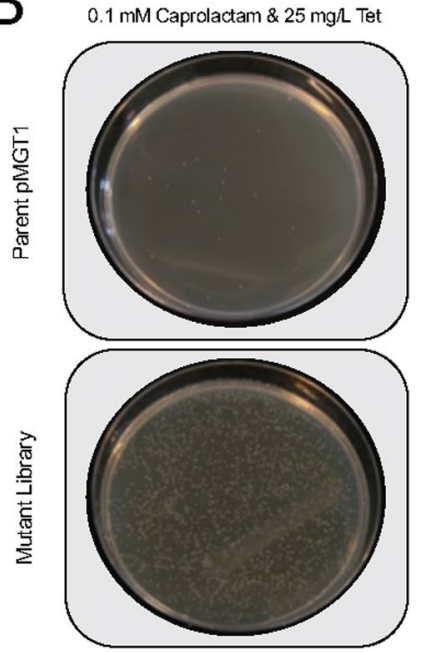

Figure 1. Selection for pMGT1 mutants with greater sensitivity. (A) Checkerboard assay showing growth rate of $E$. coli harboring pMGT1 challenged with increasing concentrations of tetracycline as a function of increasing concentrations of the inducer caprolactam. (B) Results from plate selections of the mutant pMGT1 library plated on LB agar with $0.1 \mathrm{mM}$ caprolactam and $25 \mathrm{mg} / \mathrm{L}$ tetracycline compared to E. coli harboring unmutated parent pMGT1.

complex can also associate with other RepA-iteron complexes in nearby plasmids, repressing replication of both vectors $^{5}$. This "handcuffing" process acts as an effective negative control to limit replication of pSC101 to roughly $\sim 5$ copies/cell. While multiple groups have isolated RepA or RepA homolog mutants that increase the copy number of their cognate plasmid, only two have been rigorously characterized ${ }^{8,9}$. Substituting E93 of RepA for either lysine or arginine resulted in an increased copy number from $\sim 5$ copies/cell to $\sim 30$ and $\sim 240$ copies/cell, respectively ${ }^{10}$. The authors hypothesized that these mutations disrupted the ability of RepA to dimerize, and hence the ability of the protein to control the copy number. The authors further demonstrated that these mutant origins were stable over many generations, and still compatible with ColE1 plasmids. While the authors created cloning vectors based on the high-copy origin variants, they did not rigorously characterize the effects that these elevated copy numbers had on heterologous gene expression or host growth. Deeper characterization of these altered origins may give greater insight into their usefulness as tools for synthetic biology and metabolic engineering.

In a recent paper, we described the development of a transcription factor based biosensor for industrially important lactams, mediated by the $\operatorname{araC}$-family protein $\mathrm{ChnR}^{11}$. In an attempt to select for mutations that increased the sensitivity of this system to a series of potential ligands, we isolated multiple mutations in the pSC101 origin of replication localized to the repA gene that increased copy number. Many of these mutations were distinct from mutations that have previously been described to increase the copy number of the pSC101 origin or other plasmids replicated via RepA homologs. In this study, we provide insight into the function of some of these mutated loci within the repA gene, characterize the effect that the resulting mutated origins have on a protein expressed from cognate plasmids, and determine if multiple mutations can be combined additively to affect copy number.

\section{Results}

Isolation of pSC101 origin mutants that result in higher copy number. Previous work has demonstrated that ChnR may be a useful biosensor for the detection of a myriad of industrially relevant compounds, such as valerolactam and caprolactam ${ }^{11,12}$. While $\mathrm{ChnR}$ can detect these molecules, the detection limits are well above what can currently be produced via metabolic engineering of microbial cells ${ }^{13}$. To improve the sensitivity of this transcription factor, we created a system that allowed us to select for ChnR activation through the expression of tetracycline resistance. Previously, tetracycline resistance has been demonstrated to be an excellent reporter to develop selection-based screens for bacterial transcription factors ${ }^{14}$. Plasmid pMGT1 (Supplementary Figure 1) controls the expression of tet $A$ via $\mathrm{ChnR}$, which can be activated through induction via caprolactam or other ligands in a dose-dependent manner (Fig. 1A). To generate genetic diversity within this system we created a mutagenized plasmid library by serial passaging of pMGT1 in the commercial E. coli mutator strain XL1-Red. After the mutated plasmid library was isolated and retransformed into E. coli DH10B, we used an agar plate-based checkerboard assay to identify mutants that were more sensitive to four potential ChnR ligands: caprolactam, bromocyclohexane, $\gamma$-nonalactone, and $\delta$-undecalactone (Supplementary Figure 2 ). For all putative ligands, the mutagenized library had a higher number of surviving colonies on agar plates supplemented with lower levels of inducers and higher levels of tetracycline than the unmutagenized parent plasmid (Fig. 1B).

Thirty plasmids from each ligand were isolated and fully re-sequenced to identify potential mutations. Of the 120 plasmids prepared, 114 were successfully re-sequenced. Within these 114 plasmids, 112 contained mutations relative to wild-type pMGT1 at 59 unique genetic loci. Interestingly, $73 \%$ of all mutations were localized within 


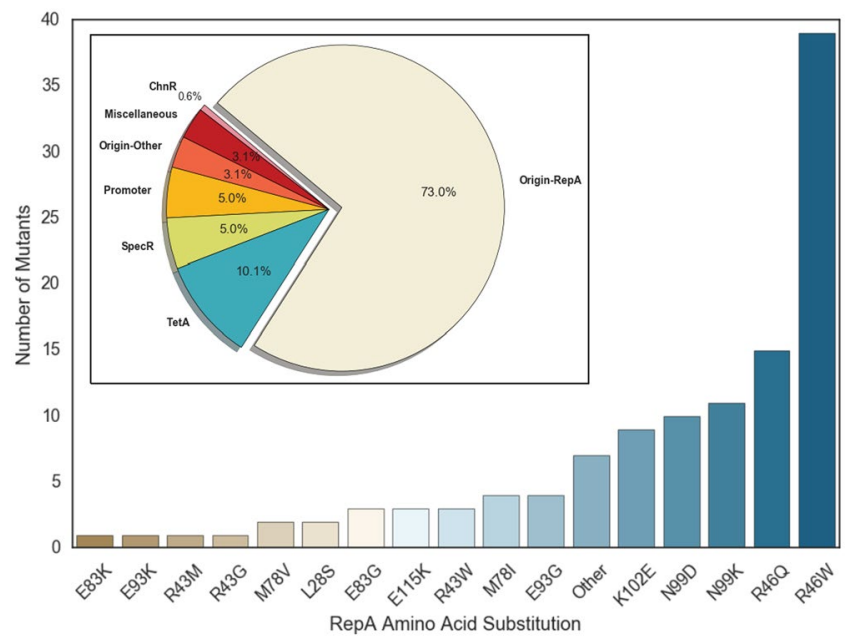

Figure 2. Distribution of mutants obtained from plate-based selections. Inset: Distribution of locations of mutations within the plasmid pMGT1. Histogram showing the frequency of selected amino acid substitutions found within RepA in re-sequenced plasmids.

the RepA protein coding sequence of the pSC101 origin, while only $0.6 \%$ of the mutations were in $\operatorname{chnR}$ itself (Fig. 2 inset). Upon further inspection, every plasmid that contained a mutation had at least one mutation within repA. Despite the small number of mutations identified within $c h n R$ itself, the abundance of mutations within repA was remarkable. Within our re-sequenced plasmids, we identified 116 mutations in repA that corresponded to 22 unique amino acid substitutions at 14 unique amino acid sites. Of the 22 unique amino acid substitutions, 13 occurred more than once (Fig. 2). Forty-six percent of all repA mutations occurred at R46, while $18 \%$ of all mutations occurred at N99. Overall, $88 \%$ of all mutations in repA resulted in a substitution of a charged amino acid. Previous reports have shown that mutations shown to increase the copy number of the pSC101 origin are often the result of amino acid substitutions that putatively disrupt dimerization of the RepA protein ${ }^{9}$. A higher copy origin would explain the increased ligand sensitivity found in our plate-based selection, as well as the apparent bias towards charged amino acid mutations, which may participate in protein-protein interactions. Therefore, we hypothesized that many of the amino acid positions that we identified contribute to the dimerization-and corresponding copy number regulation- of the pSC101 origin.

Isolated mutations in the RepA protein are predicted to lie within the dimerization interface. In order to infer the structural position of the mutated residues identified in our screen, we generated a homology model of the homodimer RepA based on the crystal structure of the homologous protein RepE, the protein that controls the replication of the mini-F plasmid (PDB ID: 2Z9O) (Fig. 3A) ${ }^{7}$. The RepE homodimer functions as a repressor while the monomer acts as a replication initiator. Though RepA shares only $19 \%$ sequence identity to RepE, the RepA homology model displayed a very similar fold with the RepE protein structure, having a root-mean-square deviation (RMSD) of $3.12 \AA$ throughout the entire polypeptide backbone (Fig. 3A).

The majority of the identified mutations were located at or near the dimerization domain of RepA (Fig. 3B-D). Two of the most common mutations identified in our study, N99 and R46, were located close to the intermolecular $\beta$-sheets near the dimerization interface (Fig. 3B and Supplementary Figure 3). A potential interaction can be observed between N99 residue from monomer A and the neighboring N99 residue from monomer B (Supplementary Figure 3). In addition, several other mutants point to potential electrostatic interactions at the dimer interface. Residues E93 and K102 are located at the intermolecular $\beta$-sheets in the dimerization interface and are close enough for potential electrostatic integrations (Fig. 3C). A similar scenario can be observed between E115 and R43 (Fig. 3D). All the charged residues mentioned are consistent with residues that are also present in the RepE dimerization interface ${ }^{7}$. In RepE, the dimerization interface is mainly formed by intermolecular $\beta$-sheets with several key interactions of charged residues. A similar residue interaction is observed in the RepA homology model. Based on these observations, the majority of the mutants that we isolated appear likely to be involved in dimerization, either through stabilizing secondary structure at the dimerization interface, or through direct electrostatic interactions.

Mutations at the RepA dimerization interface result in a variety of plasmid copy numbers. Of the 14 unique mutated amino acid residues within RepA that we identified in our screen, only one has been rigorously characterized- E93 ${ }^{10}$. Peterson et al. showed that an E93K mutation yielded a copy number of $\sim 30$ copies/ cell, while an E93R mutation resulted in a copy number of $\sim 240$ copies/cell ${ }^{10}$. To probe how our newly identified mutations impacted copy number, we created 10 amino acid substitutions at 8 amino acid residues within the RepA coding sequence of plasmid pBbS8k-RFP, chosen either by the frequency of occurrence in our screen or predicted location at the dimerization interface based on our homology model. The pBbS8k-RFP plasmid is a Bglbrick family plasmid that contains a pSC101 origin, as well as the gene encoding the red fluorescence protein (RFP) under the control of an araBAD promoter $^{1}$. The copy number of wild-type pBbS8k-RFP, in addition to that 

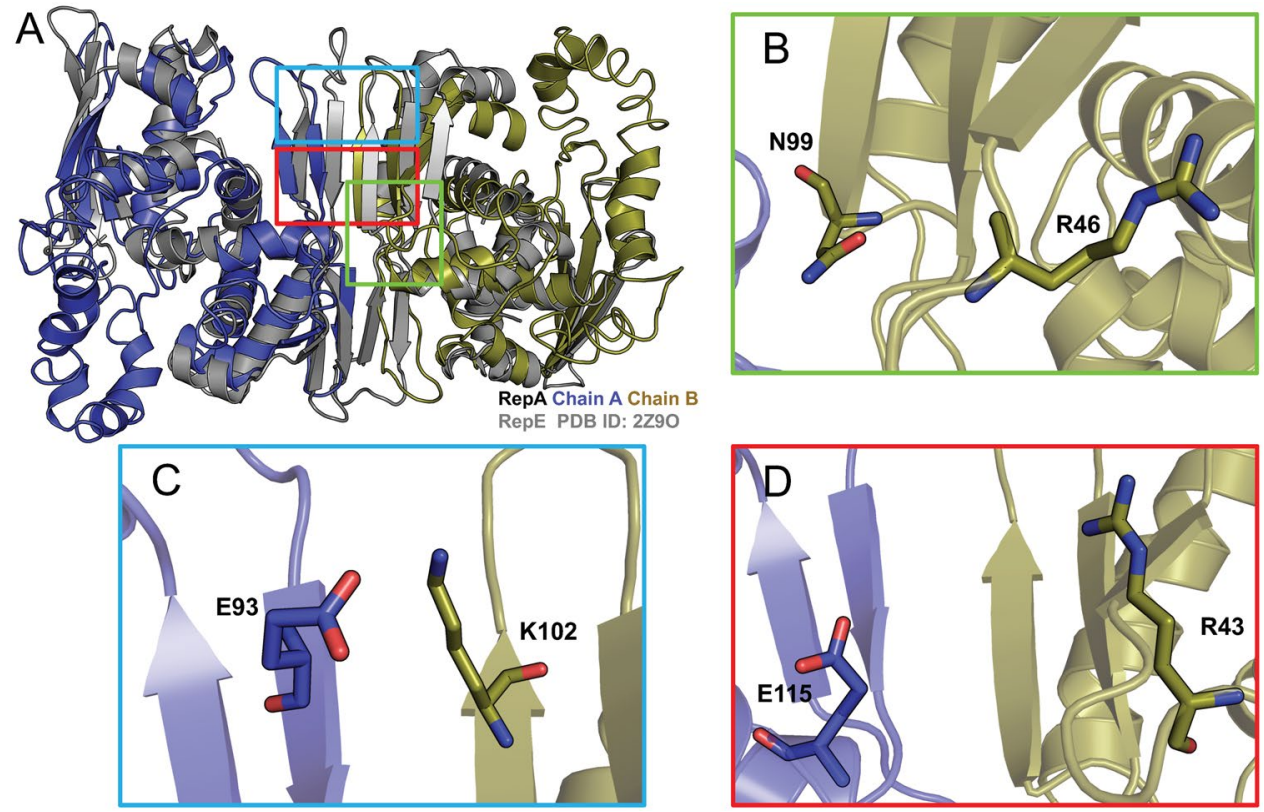

Figure 3. Homology model of RepA homodimer (shown in blue and gold) based on the RepE structure (shown in gray). (A) Full protein dimer. (B) Zoomed in view of N99 and R46 residues, the two most commonly isolated RepA mutants. (C) Potential electrostatic interaction between residues E93 and K102. (D) Potential electrostatic interaction between residues E115 and R43.

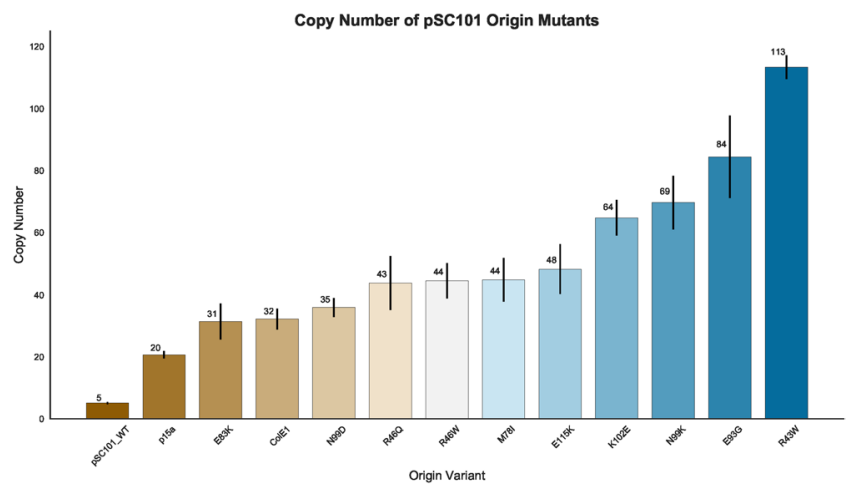

Figure 4. Copy number of control plasmids and pSC101 RepA mutants as determined by qPCR. Error bars represent $95 \%$ CI $(n=3)$.

of all ten of our selected mutated variants, was determined by qPCR in the absence of arabinose. Additionally, the copy number of pBbA8k-RFP and pBbE8k-RFP, containing a p15a and ColE1 origin, respectively, were also calculated. All ten mutations tested showed increased copy numbers ranging from $\sim 31$ to $\sim 113$ copies/cell (Fig. 4). Mutations E83K and N99D yielded copy numbers of $\sim 31$ and $\sim 35$ copies/cell, respectively, similar to that of the ColE1 origin of replication ( 32 copies/cell). Mutations R46Q, R46W, M78I, and E115K all had roughly equivalent copy numbers of approximately 45 copies/cell, while mutations K102E, N99K, E93G, and R43W all yielded higher copy numbers ranging from $\sim 64$ to $\sim 113$ copies/cell (Fig. 4 ).

We observed, as have other groups ${ }^{10}$, that the nature of the substitution within RepA can dramatically affect the resulting copy number. Two mutations at N99, N99D and N99K, resulted in markedly different copy numbers ( $\sim 35$ and $\sim 69$ copies/cell). Similarly, previous work has shown that mutations E93K and E93R raise the copy number to $\sim 30$ and $\sim 240$ copies/cell, respectively, while a E93G mutation resulted in a copy number of $\sim 84$ copies/cell ${ }^{10}$. However, in some cases multiple mutations at the same residue yielded similar copy numbers, such as mutations R46Q and R46W, which both resulted in pSC101 variants with $\sim 45$ copies/cell. From these results, it appears that a wide range of copy numbers can be obtained from a variety of repA mutations. However, whether or not these origin variants result in useful protein expression remained to be determined.

Copy number in pSC101 repA mutants does not correlate with expression of RFP. Though previous work has characterized the copy number of pSC101-derived mutants, the effect these mutations have on heterologous protein expression has not been similarly tested ${ }^{10}$. To better understand this connection, we performed 


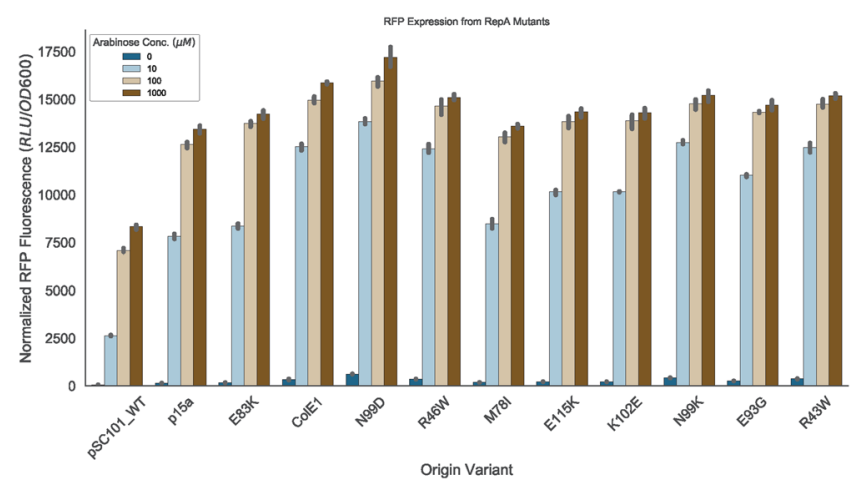

Figure 5. Production of RFP as a function of plasmid copy number and inducer concentration. Expression of $\mathrm{RFP}$ as measured by fluorescence normalized to $\mathrm{OD}_{600}$ following induction from the araBAD promoter with increasing concentrations of arabinose induction. Error bars represent $95 \% \mathrm{CI}(n=3)$. Plasmids are ordered by increasing copy number.

a series of fluorescence protein expression assays to gauge whether copy number was correlated with increased RFP production from our vectors. To achieve this, we transformed our mutant and wild-type plasmids into $E$. coli DP10, which has the arabinose transporter, encoded by araFGH, under control of a constitutive promoter ${ }^{15}$ to allow for inducible expression from the araBAD promoter, and evaluated expression of RFP at increasing concentrations of arabinose (Fig. 5). As expected, all mutants had higher levels of RFP expression than wild-type pSC101 at all concentrations of arabinose tested. However, there was no overall correlation between copy number and RFP expression at any concentration of arabinose (Supplementary Figure 4). In fact, the highest expression of RFP achieved was from the N99D mutant, which has a copy number of $\sim 35$ copies/cell, similar to that of the ColE1 origin. Of the mutants with copy numbers below 35 copies/cell, mutant E83K (copy number of $\sim 31$ copies/ cell) showed slightly higher expression than the wild-type p15a origin, which had a copy number of $\sim 20$ copies/ cell. These two mutated origins, N99D and E83K, may prove useful in future metabolic engineering efforts.

Mutant pSC101 origins remain compatible with other commonly used plasmid origins of replication. In order for higher copy variants of the pSC101 origin to be useful in synthetic biology, they must maintain their compatibility with other commonly used origins of replication. Previous work has demonstrated that specific high-copy pSC101 mutants maintained compatibility with the pBBR origin, but did not investigate other common origins ${ }^{10}$. To interrogate compatibility more thoroughly used a previously described method wherein we transformed either a pSC101 wild-type vector or the N99D and E83K mutants, all of which encoded the kanamycin resistance marker, into strains of $E$. coli harboring either p15a, pBBR, or ColE1 origins of replication with a carbenicillin selectable marker ${ }^{10}$. Cells containing both plasmids were passaged for 60 generations while only selecting for the carbenicillin resistance marker. Compatibility was assessed every 20 generations by determining the ratio of cells that survived plating on LB Carb/Kan plates relative to LB Carb plates (Fig. 6). All pSC101 origins were maintained at greater than $93 \%$ of all cells for all origins tested, indicating that both the N99D and the E83K mutants retain compatibility with other commonly used E. coli origins.

Mutations predicted to be at the RepA dimer interface can be combined to affect copy number. While there may be limited benefit to increasing the copy number of the pSC101 origin past $~ 35$ copies/cell, we were still interested in knowing if mutations at multiple loci could be combined additively to affect copy number. Based on our homology modelling, we identified two pairs of amino acids that could potentially be involved in electrostatic interactions at the dimerization interface, E93 and K102, as well as E115 and R43. We sought to disrupt both of these interactions by creating two double mutants, K102E-E115K and R43W-E93G. As before, we determined the copy number of the double mutants by qPCR (Fig. 7). The copy number of the K102E-E115K double mutant was roughly equivalent to the sum of the copy number of its individual component mutations. However, the R43W-E93G mutant had a much higher copy number of $~ 494$ copies/cell, more than two and a half times that of its corresponding single mutants combined. This suggests that these two mutations in tandem are sufficient to severely disrupt the ability of RepA to dimerize.

While the copy number did dramatically increase in double mutant RepA proteins, neither of the double mutants was able to produce as much RFP at high arabinose concentration $(100 \mu \mathrm{M}$ and $1000 \mu \mathrm{M})$ as the N99D single mutation; nonetheless, the R43W-E93G mutant was able to produce RFP at levels greater than that of the ColE1 origin of replication at $10 \mu \mathrm{M}$ arabinose (Supplementary Figure 5A). At higher concentrations of arabinose induction both double mutants had reduced growth rates, particularly in R43W-E93G (Supplementary Figure 6A). Though there was no correlation between fluorescence and copy number within our RepA mutants, there appeared to be a correlation between copy number and maximal growth rate as arabinose concentration increased with a Pearson-R of -0.91 at $100 \mu \mathrm{M}$ arabinose and -0.87 at $1000 \mu \mathrm{M}$ arabinose (Supplementary Figures 6B-E). 


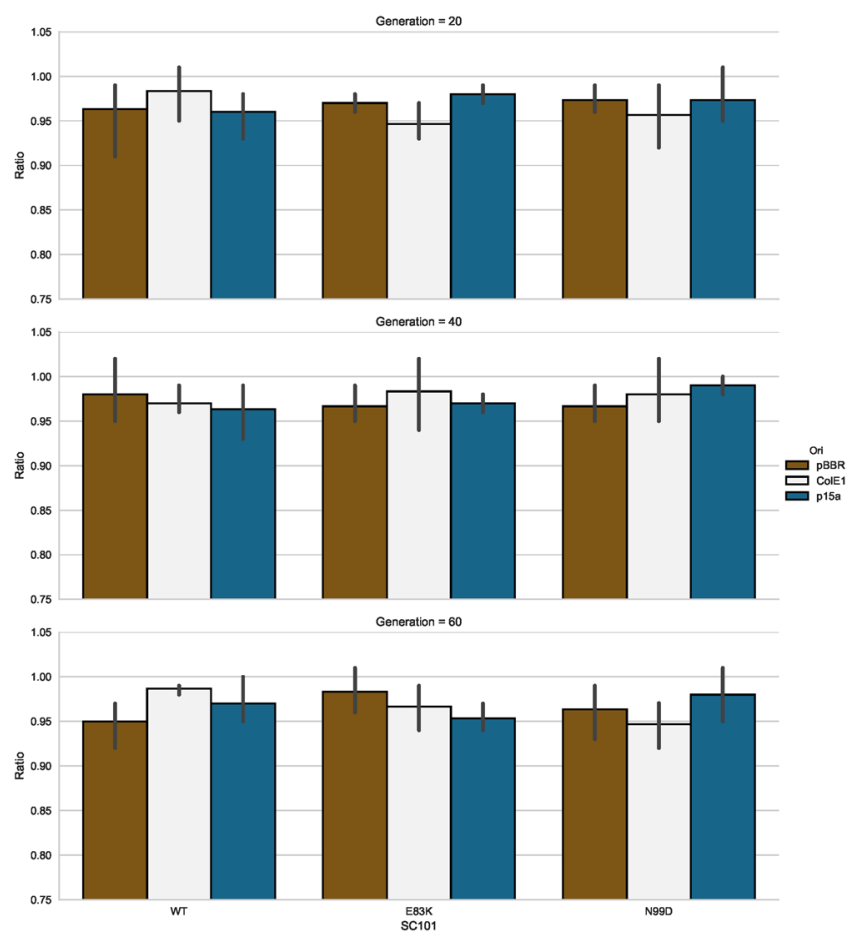

Figure 6. Plasmid compatibility of potentially useful pSC101 origin mutants with other commonly used E. coli plasmid origins. pSC101 wt and the E83K and N99D mutants encoded kanamycin resistance, while pBBR and p15a encoded carbenicillin resistance. Ratios of the number of CFUs recovered on Carb/Kan LB plates relative to the number of CFUs recovered on LB Carb alone. Error bars represent 95\% CI.

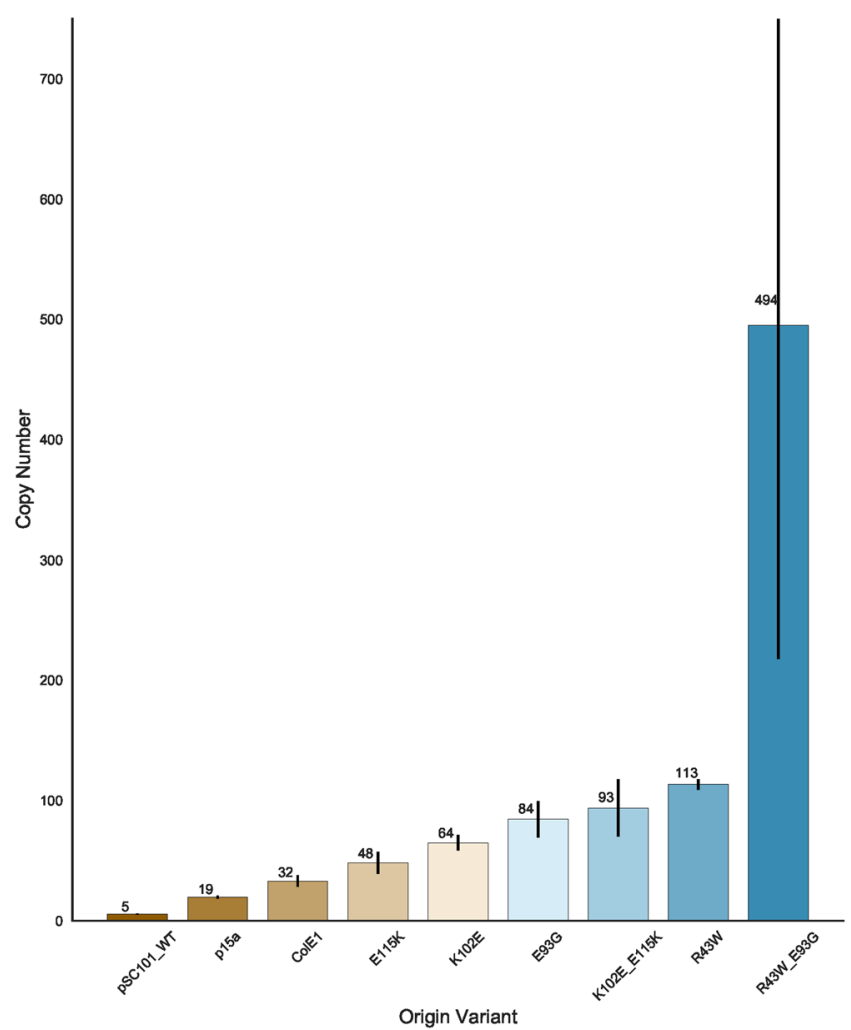

Figure 7. Copy number of control plasmids, parent RepA mutations, and double RepA mutations predicted to be involved in electrostatic interactions between monomers, as estimated by qPCR. Error bars represent $95 \%$ CI $(\mathrm{n}=3)$. 


\section{Discussion}

In this work, we isolated 10 previously undescribed RepA mutations that increase the copy number of the pSC101 origin. Single mutations resulted in copy numbers ranging from $\sim 31$ to $\sim 113$ copies/cell, compared to $\sim 5$ copies/ cell in wild-type pSC101 plasmids. The majority of these mutations are in charged amino acids that are predicted to lie on the dimerization interface of the RepA protein. Two of these mutations, R43W and E93G, could be combined to achieve a copy number of $\sim 494$ copies/cell. When these mutations were incorporated into a plasmid containing RFP under the control of an arabinose-inducible promoter, no overall correlation was observed between the copy number and fluorescence. As copy number increased, there was a corresponding decrease in growth rate, with the highest copy double mutant showing severely diminished growth at higher levels of arabinose induction.

Homology modelling suggests that many of the mutations identified in this study lie within $\beta$-sheets on the dimerization interface of the RepA protein. Structural studies of the RepA homolog, RepE which controls the copy number of the mini-F plasmid, indicated a very similar intermolecular $\beta$-sheet dimeric interface ${ }^{16}$. RepE plays an important role in controlling replication of the F plasmid origin, ori2. Monomeric RepE binds to ori2 to initiate replication, whereas RepE homodimers act as an autogenous repressor by binding to the repE operator $^{16}$. In another study, RepA was activated by the heat shock proteins DnaK and Dna ${ }^{17}$. Both DnaK and DnaJ activated RepA by converting RepA dimers into monomers in an ATP-dependent reaction and simultaneously activated oriP1 DNA binding ${ }^{17}$. Based on these observations, the majority of the mutants that we isolated appear to be involved in dimerization, either through stabilizing the secondary structure at the dimerization interface, or through direct electrostatic interactions. Each mutation in the double mutant constructs R43W-E93G or K102E-E115K is located at different regions of the dimer interface and may explain the additive effect of increase copy number. Such structural perturbations in RepA may cause a disruption in its oligomeric state and hence, plasmid copy regulation. Due to the limits of homology models and lack of a RepA crystal structure, it is difficult to reliably categorize and measure distinct residue interactions at the dimer interface. Future efforts in obtaining a crystal structure of RepA may provide further detail.

Of the mutations that we isolated in this work, E83K and N99D may be particularly useful for synthetic biology applications. Plasmids harboring the E83K mutation showed a very similar expression profile to that of a p15a expression vector, while the N99D mutant plasmid demonstrated slightly higher expression than the ColE1 derivative. Importantly, both of these mutants retain compatibility with other commonly used origins of replication in E. coli. Previous work has demonstrated that copy number mutants maintain their compatibility with ColE1 origin plasmids, making these mutants potentially valuable when engineering systems that require multiple medium or high copy vectors ${ }^{10}$. RFP expression, as measured by fluorescence, plateaued for RepA mutant-derived plasmids with copy numbers above $~ 35$ copies/cell, which can be explained in a variety of ways. While very high-copy double mutants achieved higher expression at low levels of induction $(10 \mu \mathrm{M})$, they were severely detrimental to growth at higher inducer concentration. One limitation of this study was the reliance on RFP fluorescence as a measure of expression, which may not be predictive of other proteins ${ }^{18,19}$. Future work will need to be conducted to assess the expression profiles of other proteins. The reasons for this plateau may be manifold due to mRNA stability, metabolic burden of maintaining the plasmid, or limitation of key intracellular metabolites ${ }^{4,20}$. Further work will be necessary to fully characterize why no additional expression can be obtained by increasing copy number in these systems.

As metabolic engineering and synthetic biology mature as disciplines, many groups have looked beyond model organisms as chasses for engineering efforts ${ }^{21}$. While rudimentary genetic systems exist for some of these organisms, they lack the robust tool sets that have been developed for model organisms such as Saccharomyces cerevisiae and E. coli. With relatively few high- or medium-copy vectors characterized for non-model organisms, we believe that this work could serve as a template for developing differential copy vectors derived from plasmids controlled by RepA homologs in non-model hosts. By mutagenizing repA or a repA homolog within a plasmid expressing a resistance determinant from an inducible promoter, variable copy numbers should be able to be isolated by imposing different strengths of selection for the resistance determinant. Alternatively, further structural information on RepA and its homologs may allow for more targeted engineering of dimer interactions that would allow for predictable copy numbers as a function of the strength of dimerization. Regardless of the approach taken, mutagenesis of RepA-dependent plasmids may offer a relatively easy way to develop a range of useful copy number variants in hosts that are desirable for metabolic engineering.

\section{Methods}

Media, Bacterial Growth Conditions, and Reagents. All bacterial strains are listed in Table 1. All strains and plasmids created in this work are available through the public instance of the JBEI registry (https://public-registry.jbei.org/folders/338). Bacteria were cultivated in Luria-Bertani (LB) Miller media (BD Biosciences, San Jose, CA) at $37^{\circ} \mathrm{C}$ unless otherwise noted, supplemented with kanamycin $(50 \mathrm{mg} / \mathrm{L}$, Sigma Aldrich, USA) or spectinomycin (100 mg/L, Fisher Scientific, USA) when indicated. Tetracycline hydrochloride used in plate selections was added at concentrations indicated, as were inducers bromocyclohexane, caprolactam, $\delta$-undecalactone, and $\gamma$-nonalactone. All compounds were purchased through Sigma Aldrich, USA.

DNA Manipulation. All plasmids used in this study are listed in Table 1. All primers used in this study are listed in Supplementary Table 1. Plasmids were designed using Device Editor and Vector Editor software ${ }^{22,23}$, and primers for construction of plasmids were designed using $j 5^{24}$. All plasmids were assembled via Gibson Assembly ${ }^{25}$. Plasmids were isolated using the Qiaprep Spin Miniprep kit (Qiagen, USA), and all primers were purchased from Integrated DNA Technologies (IDT, Coralville, IA).

Site Directed Mutagenesis of repA. To construct site-specific mutants, the parent plasmid pBbS8k-RFP was used as a template to amplify the entire plasmid into two PCR products. Mutations were introduced in 


\begin{tabular}{|c|c|c|c|}
\hline Strain & Notes & Reference & JBEI ID \\
\hline E. coli DH10B & & Invitrogen & \\
\hline E. coli DP10 & $\triangle a r a F G H \triangle \mathrm{P}-a r a E \mathrm{pCP} 18$-araE: for linear arabinose induction & 15 & \\
\hline E. coli XL1-Red & Commercial mutator strain & Agilent & \\
\hline Plasmid & Notes & Reference & \\
\hline pBbS8k-RFP_R43W & RepA Mutant, Kan & This work & JPUB_009625 \\
\hline pBbS8k-RFP_R46W & RepA Mutant, Kan & This work & JPUB_009627 \\
\hline pBbS8k-RFP_R46Q & RepA Mutant, Kan & This work & JPUB_009629 \\
\hline pBbS8k-RFP_M78I & RepA Mutant, Kan & This work & JPUB_009631 \\
\hline pBbS8k-RFP_E83K & RepA Mutant, Kan & This work & JPUB_009633 \\
\hline pBbS8k-RFP_E93G & RepA Mutant, Kan & This work & JPUB_009635 \\
\hline pBbS8k-RFP_N99D & RepA Mutant, Kan & This work & JPUB_009637 \\
\hline pBbS8k-RFP_N99K & RepA Mutant, Kan & This work & JPUB_009639 \\
\hline pBbS8k-RFP_K102E & RepA Mutant, Kan & This work & JPUB_009641 \\
\hline pBbS8k-RFP_E115K & RepA Mutant, Kan & This work & JPUB_009643 \\
\hline pBbS8k-RFP_R43W_E93G & RepA Double Mutant, Kan & This work & JPUB_009645 \\
\hline pBbS8k-RFP_K102E_E115K & RepA Double Mutant, Kan & This work & JPUB_009647 \\
\hline pBbS8k-RFP & AraC Expression Vector, pSC101 Ori, Kan & 1 & \\
\hline pBbA8k-RFP & AraC Expression Vector, p15a Ori, Kan & 1 & \\
\hline pBbE8k-RFP & AraC Expression Vector, ColE1 Ori, Kan & 1 & \\
\hline pBbE8a-RFP & AraC Expression Vector, ColE1 Ori, Amp & 1 & \\
\hline pBbB8a-RFP & AraC Expression Vector, BBR Ori, Amp & 1 & \\
\hline pBbS8a-RFP & AraC Expression Vector, p15a Ori, Amp & 1 & \\
\hline pMGT1 & ChnR Biosensor with TetA Reporter, pSC101 Ori, Spec & This work & JPUB_009650 \\
\hline pBbSlactamC-mCherry (star) & ChnR Biosensor with mCherry Reporter, pSC101 Ori, Spec & 11 & \\
\hline
\end{tabular}

Table 1. All strains and plasmids used in this study. Strains and plasmids created in this study can be found at https://public-registry.jbei.org/folders/338.

primers that bound within the repA gene. Plasmids were assembled from these products via Gibson assembly, and mutations were confirmed via Sanger sequencing (Quintara Biosciences, Albany, CA).

Creation of a Mutagenesis Library of pMGT1. Mutagenesis of the lactam-biosensing pMGT1 tetA reporter plasmid was performed in vivo in the E. coli mutator strain XL1-Red (Agilent Technologies, Santa Clara, CA). The plasmid was transformed into XL1-Red competent cells via electroporation and plated on LB agar plates supplemented with spectinomycin. After incubating at $37^{\circ} \mathrm{C}$ overnight, $\sim 2000$ colonies were scraped and were then resuspended in $50 \mathrm{~mL}$ of LB medium supplemented with spectinomycin and grown overnight at $37^{\circ} \mathrm{C}$. Afterward, $1 \mathrm{~mL}$ of the culture was removed and stored at $-80^{\circ} \mathrm{C}$ for future plasmid isolation. This overnight was then used to inoculate $500 \mu \mathrm{L}$ of LB spectinomycin 1:100 in each well of a 96-well deep-well plate (VWR, USA). Passaging of cultures was done in a 96-well format to help prevent selective sweeps by non-mutator phenotypes of XL1-Red that may arise. Passages were performed for five days every 20 hours, by inoculating fresh media in 96-well plates via a pin-replicator. Each day after passaging, culture from all wells was pooled and frozen for subsequent plasmid isolation. Plasmids from all passages were isolated and pooled and then transformed into E. coli MegaX DH10B T1R Electrocomp Cells (Invitrogen, Carlsbad, CA) via electroporation, yielding roughly twelve million transformants. These transformants were pooled and frozen at $-80^{\circ} \mathrm{C}$ for later use.

Selection for Mutants. To select for mutations that were more sensitive to ligands of interest, E. coli $\mathrm{DH} 10 \mathrm{~B}$ cultures containing either the mutagenized plasmids or the parent plasmid were grown overnight in LB medium supplemented with spectinomycin, and then plated on LB agar plates that contained various concentrations of inducers and tetracycline. Inducers used to select for mutations were bromocyclohexane, caprolactam, $\delta$-undecalactone, and $\gamma$-nonalactone. Plates contained either $1000 \mu \mathrm{M}, 500 \mu \mathrm{M}, 100 \mu \mathrm{M}$, or $0 \mu \mathrm{M}$ of each inducer. Plates contained either $50 \mathrm{mg} / \mathrm{L}, 25 \mathrm{mg} / \mathrm{L}, 12.5 \mathrm{mg} / \mathrm{L}$, or $0 \mathrm{mg} / \mathrm{L}$ tetracycline (see Supplementary Figure 2). Colonies from library plates that were able to grow on higher concentrations of tetracycline at lower concentrations of inducer after overnight incubation were considered for further analysis. Thirty plasmids selected from each inducer were purified and re-sequenced to identify the location of mutations.

Re-sequencing Plasmids via Next-Generation Sequencing. Nextera libraries were constructed using the Illumina Nextera XT Library Preparation Kit and the Nextera XT Index Kit v2 (Illumina, San Diego, CA). Liquid transfers were carried out on the BioMek NX, Biomek Fx (Beckman Coulter, Indianapolis, IN), and Labcyte Echo 550 acoustic liquid dispensing system (Labcyte, Sunnyvale, CA). During tagmentation, DNA was fragmented by transposases, and transposon terminal sequences were appended as adapters. Unique barcode combinations were added during PCR amplification. The tagmentation and PCR amplification steps were performed with several modifications to the standard Nextera protocol: 1) all steps were performed in a 
single 384-well PCR plate, 2) the tagmentation reaction volume was reduced to $1 \mu \mathrm{L}$ using the Echo 550, and 3) a heat-kill step $\left(15\right.$ minutes at $\left.70^{\circ} \mathrm{C}\right)$ was added to circumvent the need for purification after tagmentation. For the qPCR step, $7.5 \mu$ l SsoAdvanced Universal SYBR Green Supermix $(2 \times)$ (Bio-Rad, Hercules, CA) and $5.5 \mu \mathrm{l}$ Nuclease free water (Thermo Fisher Scientific, Waltham, MA) were added to the tagmentation reaction. The end point fluorescence values from qPCR on a CFX384 (Bio-Rad, Hercules, CA) were used to normalize concentrations using the Echo 550. This enabled Ampure XP bead purification (A63880, Beckman Coulter, Indianapolis, IN) of the pooled library in a single tube. The purified library was quantified using the Qubit DNA HS assay (Invitrogen, Carlsbad, CA), and the fragment size profile was determined using the Bioanalyzer 2100 (Agilent Technologies, Santa Clara, CA). Sequencing was performed on the MiSeq (Illumina, San Diego, CA) using $2 \times 300$ cycles. MiSeq reads were demultiplexed using the embedded MiSeq Reporter (MSR) software. Read mapping and variant calling were performed using $\mathrm{BWA}^{26}$, and $\mathrm{GATK}^{27}$.

Isolation of Total DNA. Isolation of total DNA was performed via the method employed by Lee et al., with minor changes ${ }^{1,28,29}$. Overnight cultures harboring plasmids of interest were inoculated 1:100 in LB kanamycin. Cultures were grown to an $\mathrm{OD}_{600}$ of $\sim 0.3$, and then $2 \mathrm{~mL}$ of culture was pelleted and frozen at $-80^{\circ} \mathrm{C}$. Cell pellets were resuspended in $400 \mu \mathrm{L}$ of $50 \mathrm{mM}$ Tris $/ 50 \mathrm{mM}$ EDTA, $\mathrm{pH} 8$, and then permeabilized with $8 \mu \mathrm{L}$ of $50 \mathrm{mg} / \mathrm{mL}$ lysozyme (Sigma Aldrich, USA) in $10 \mathrm{mM}$ Tris/1 mM EDTA, $\mathrm{pH} 8$, followed by incubation at $37^{\circ} \mathrm{C}$ for $30 \mathrm{~min}-$ utes. Cells were then lysed by the addition of $8 \mu \mathrm{L}$ of $5 \%$ SDS and $8 \mu \mathrm{L}$ of $20 \mathrm{mg} / \mathrm{mL}$ Proteinase K solution (Qiagen, USA), after which they were mixed with a syringe with a 21 -gauge, 1.5 -inch needle, followed by incubation at $50^{\circ} \mathrm{C}$ for 30 minutes. After inactivating Proteinase $\mathrm{K}$ by incubation at $75^{\circ} \mathrm{C}$ for $10 \mathrm{~min}, 2 \mu \mathrm{L}$ of $100 \mathrm{mg} / \mathrm{mL} \mathrm{RNase}$ A solution (Qiagen, USA) was added and then incubated at $37^{\circ} \mathrm{C}$ for 30 minutes. Total DNA was extracted via a phenol:chloroform extraction, followed by ethanol precipitation as described previously ${ }^{1}$. Isolated gDNA was digested for 6 hours at $37^{\circ} \mathrm{C}$ with Fast Digest EcoRI (Thermo Fisher Scientific, Waltham, MA).

Estimation of Copy Number via qPCR. Estimation of copy number was also carried out in a manner similar to that of Lee et al., with minor changes ${ }^{1,8,28}$. Primer sets specific to the neomycin phosphotransferase II (nptII) gene (forward: GCGTTGGCTACCCGTGATAT, reverse: AGGAAGCGGTCAGCCCAT) and 16S rDNA gene (forward: CCGGATTGGAGTCTGCAACT, reverse: GTGGCATTCTGATCCACGATTAC) were used for real-time qPCR. E. coli DH10B gDNA was used for calibrating the 16S rDNA primers, while E. coli BW25113 with a single copy of $n p t I I$ integrated into the genome was used to calibrate the $n p t I I$ primers. Real-time qPCR was conducted on a BioRad CFX with 96-well reaction blocks in the presence of SYBR Green under the following conditions: $1 \times$ ssoAdvance SYBR Green Supermix (BioRad, Hercules, CA) and $150 \mathrm{nM} n p t I I$ or $500 \mathrm{nM} 16 \mathrm{~S}$ primers, in $20 \mu \mathrm{L}$ reactions. Real-time qPCR cycling parameters were $95^{\circ} \mathrm{C}$ for 3 minutes, followed by 40 cycles of 30 seconds at $95^{\circ} \mathrm{C}$, and 45 seconds at $60^{\circ} \mathrm{C}$. Threshold cycles (Ct) were determined with CFX Manager (BioRad, Hercules, CA) software for all samples.

Growth Rate and Fluorescence Assays. Growth rates of bacterial strains were estimated through a microplate reader kinetic assay. Strains were grown overnight from glycerol stocks in LB liquid medium with kanamycin, then diluted 1:100 into fresh LB media with kanamycin amended with either $1000 \mu \mathrm{M}, 100 \mu \mathrm{M}, 10 \mu \mathrm{M}$, or $0 \mu \mathrm{M}$ arabinose in 96-well plates (Falcon, 353072). Plates were sealed with a gas-permeable microplate adhesive film (VWR, USA), and then optical density and fluorescence were monitored for 22 hours in an Infinite F200 Pro (Tecan Life Sciences, San Jose, CA) plate reader at $30^{\circ} \mathrm{C}$. Optical density was measured at $600 \mathrm{~nm}$, while fluorescence was measured using an excitation wavelength of $575 \mathrm{~nm}$ and an emission wavelength of $620 \mathrm{~nm}$ with a manually set gain of 35 . In between reads, the plate was shaken at a linear amplitude of $6 \mathrm{~mm}$.

To measure RFP production from plasmids, fluorescence measurements were obtained from single time points of cells grown in deep-well 96-well plates. Cells were grown in $500 \mu \mathrm{L}$ of LB medium with kanamycin supplemented with either $1000 \mu \mathrm{M}, 100 \mu \mathrm{M}, 10 \mu \mathrm{M}$, or $0 \mu \mathrm{M}$ arabinose. Plates were sealed with AeraSeal film (Excel Scientific, AC1201-02) and grown for 22 hours at $30^{\circ} \mathrm{C}$ on a $200 \mathrm{rpm}$ shaker rack. After incubation, $100 \mu \mathrm{L}$ from each well was aliquoted into a black, clear-bottom 96-well plate and fluorescence was measured with an Infinite F200 Pro (Tecan Life Sciences, San Jose, CA) plate reader. Optical density was measured at $600 \mathrm{~nm}\left(\mathrm{OD}_{600}\right)$, while fluorescence was measured using an excitation wavelength of $575 \mathrm{~nm}$ and an emission wavelength of $620 \mathrm{~nm}$ with a manually set gain of 35 .

Plasmid Compatibility Assay. Mutant plasmids pBbS8k-RFP_E83K and pBbS8k-RFP_N99D were cotransformed into E. coli DH10B with a BglBrick family plasmid containing the gene encoding RFP under the control of an $\operatorname{araBAD}$ promoter, and a carbenicillin resistance cassette. The origin of replication for the latter vector was either p15a, ColE1, or pBBR1. To test plasmid stability and compatibility, the transformants were grown overnight in LB containing $100 \mu \mathrm{g} / \mathrm{mL}$ carbenicillin. Each overnight culture was considered to be the result of 10 generations of growth. Each overnight culture was diluted by a factor of 1:1,000 ( $3 \mu \mathrm{L}$ into $3 \mathrm{~mL}$ ) and grown again for a total of 60 generations, i.e. six days. Every other day, after 20 generations of growth, the cultures were diluted 1:50,000 and plated onto LB plates containing only carbenicillin, as well as plates containing both carbenicillin and kanamycin. Colonies were then counted after incubation overnight. Stability was assessed as the ratio between the number of colonies present on the carb/kan plates and the number of colonies present on the carb only plates.

RepA Structural Modelling. The RepA homology model was generated using the structure prediction software I-TASSER (https://zhanglab.ccmb.med.umich.edu/) ${ }^{30,31}$. The internal C-score scoring function in I-TASSER was utilized to select the best homology model. The known homodimeric RepE protein structure (PDB ID: 2Z9O) 
was utilized to generate a homodimeric structure of the RepA homology model. The RepA homodimeric model was energy minimized using CHIMERA ${ }^{32}$. All figures related to the RepA homology model were visualized and generated by PyMOL (The PyMOL Molecular Graphics System, Version 1.8 Schrödinger, LLC.).

Data Visualization and Statistical Analysis. All data were analyzed using custom Python scripts. All graphs were visualized using either Seaborn or Matplotlib. Calculation of $95 \%$ confidence intervals was conducted via the Scipy library.

\section{References}

1. Lee, T. S. et al. BglBrick vectors and datasheets: A synthetic biology platform for gene expression. J Biol Eng 5, 12 (2011).

2. George, K. W. et al. Correlation analysis of targeted proteins and metabolites to assess and engineer microbial isopentenol production. Biotechnol Bioeng 111, 1648-1658 (2014).

3. Alonso-Gutierrez, J. et al. Metabolic engineering of Escherichia coli for limonene and perillyl alcohol production. Metab Eng 19, 33-41 (2013).

4. Carrier, T., Jones, K. L. \& Keasling, J. D. mRNA stability and plasmid copy number effects on gene expression from an inducible promoter system. Biotechnol Bioeng 59, 666-672 (1998).

5. Furuno, S., Watanabe-Murakami, Y., Takebe-Suzuki, N. \& Yamaguchi, K. Negative control of plasmid pSC101 replication by increased concentrations of both initiator protein and iterons. J Gen Appl Microbiol 46, 29-37 (2000).

6. Chattoraj, D. K. Control of plasmid DNA replication by iterons: no longer paradoxical. Mol Microbiol 37, 467-476 (2000).

7. Nakamura, A., Wada, C. \& Miki, K. Structural basis for regulation of bifunctional roles in replication initiator protein. Proc Natl Acad Sci USA 104, 18484-18489 (2007).

8. Wadood, A., Dohmoto, M., Sugiura, S. \& Yamaguchi, K. Characterization of copy number mutants of plasmid pSC101. J Gen Appl Microbiol 43, 309-316 (1997).

9. Giraldo, R. \& Fernández-Tresguerres, M. E. Twenty years of the pPS10 replicon: insights on the molecular mechanism for the activation of DNA replication in iteron-containing bacterial plasmids. Plasmid 52, 69-83 (2004)

10. Peterson, J. \& Phillips, G. J. New pSC101-derivative cloning vectors with elevated copy numbers. Plasmid 59, 193-201 (2008).

11. Zhang, J. et al. Development of a Transcription Factor-Based Lactam Biosensor. ACS Synth Biol 6, 439-445 (2017).

12. Steigedal, M. \& Valla, S. The Acinetobacter sp. chnB promoter together with its cognate positive regulator ChnR is an attractive new candidate for metabolic engineering applications in bacteria. Metab Eng 10, 121-129 (2008).

13. Chae, T. U., Ko, Y.-S., Hwang, K.-S. \& Lee, S. Y. Metabolic engineering of Escherichia coli for the production of four-, five- and sixcarbon lactams. Metab Eng 41, 82-91 (2017).

14. Dietrich, J. A., Shis, D. L., Alikhani, A. \& Keasling, J. D. Transcription factor-based screens and synthetic selections for microbial small-molecule biosynthesis. ACS Synth Biol 2, 47-58 (2013).

15. Pitera, D. J., Paddon, C. J., Newman, J. D. \& Keasling, J. D. Balancing a heterologous mevalonate pathway for improved isoprenoid production in Escherichia coli. Metab Eng 9, 193-207 (2007).

16. Ishiai, M., Wada, C., Kawasaki, Y. \& Yura, T. Replication initiator protein RepE of mini-F plasmid: functional differentiation between monomers (initiator) and dimers (autogenous repressor). Proc Natl Acad Sci USA 91, 3839-3843 (1994).

17. Wickner, S., Hoskins, J. \& McKenney, K. Monomerization of RepA dimers by heat shock proteins activates binding to DNA replication origin. Proc Natl Acad Sci USA 88, 7903-7907 (1991).

18. Reisbig, M. D., Hossain, A. \& Hanson, N. D. Factors influencing gene expression and resistance for Gram-negative organisms expressing plasmid-encoded ampC genes of Enterobacter origin. J Antimicrob Chemother 51, 1141-1151 (2003).

19. Strack, R. L. et al. A noncytotoxic DsRed variant for whole-cell labeling. Nat Methods 5, 955-957 (2008).

20. Smolke, C. D. \& Keasling, J. D. Effect of copy number and mRNA processing and stabilization on transcript and protein levels from an engineered dual-gene operon. Journal of Biochemical and Microbiological Technology and Engineering 78, 412-424 (2002).

21. Loeschcke, A. \& Thies, S. Pseudomonas putida-a versatile host for the production of natural products. Appl Microbiol Biotechnol 99, 6197-6214 (2015).

22. Chen, J., Densmore, D., Ham, T. S., Keasling, J. D. \& Hillson, N. J. DeviceEditor visual biological CAD canvas. J Biol Eng 6, 1 (2012).

23. Ham, T. S. et al. Design, implementation and practice of JBEI-ICE: an open source biological part registry platform and tools. Nucleic Acids Res 40, e141 (2012).

24. Hillson, N. J., Rosengarten, R. D. \& Keasling, J. D. j5 DNA assembly design automation software. ACS Synth Biol 1, 14-21 (2012).

25. Gibson, D. G. et al. Enzymatic assembly of DNA molecules up to several hundred kilobases. Nat Methods 6, 343-345 (2009).

26. Li, H. Aligning sequence reads, clone sequences and assembly contigs with BWA-MEM. arXiv (2013).

27. McKenna, A. et al. The Genome Analysis Toolkit: a MapReduce framework for analyzing next-generation DNA sequencing data. Genome Res 20, 1297-1303 (2010).

28. Mason, G., Provero, P., Vaira, A. M. \& Accotto, G. P. Estimating the number of integrations in transformed plants by quantitative real-time PCR. BMC Biotechnol 2, 20 (2002).

29. Pushnova, E. A., Geier, M. \& Zhu, Y. S. An easy and accurate agarose gel assay for quantitation of bacterial plasmid copy numbers. Anal Biochem 284, 70-76 (2000).

30. Roy, A., Kucukural, A. \& Zhang, Y. I-TASSER: a unified platform for automated protein structure and function prediction. Nat Protoc 5, 725-738 (2010).

31. Zhang, Y. I-TASSER server for protein 3D structure prediction. BMC Bioinformatics $\mathbf{9 ,} 40$ (2008).

32. Pettersen, E. F. et al. UCSF Chimera-a visualization system for exploratory research and analysis. J Comput Chem 25, 1605-1612 (2004).

\section{Acknowledgements}

The authors would like to thank Tristan de Rond, Catharine Adams, and Robert Haushalter for their helpful discussions in preparing this manuscript. We would also like to thank the UC Berkeley SMART and SURF programs, as well as the Rose Hills Foundation for providing support for N.S. to conduct summer research. This work was part of the DOE Joint BioEnergy Institute (https://www.jbei.org) supported by the U.S. Department of Energy, Office of Science, Office of Biological and Environmental Research, and was part of the Agile BioFoundry (http:// agilebiofoundry.org) supported by the U.S. Department of Energy, Energy Efficiency and Renewable Energy, Bioenergy Technologies Office, through contract DE-AC02-05CH11231 between Lawrence Berkeley National Laboratory and the U.S. Department of Energy. The views and opinions of the authors expressed herein do not necessarily state or reflect those of the United States Government or any agency thereof. Neither the United States Government nor any agency thereof, nor any of their employees, makes any warranty, expressed or implied, or assumes any legal liability or responsibility for the accuracy, completeness, or usefulness of any information, apparatus, product, or process disclosed, or represents that its use would not infringe privately owned rights. 


\section{Author Contributions}

Conceptualization, M.G.T., and N.S.; Methodology, M.G.T., N.S., J.F.B., and N.J.H.; Investigation, M.G.T., N.S., N.K., J.F.B., M.W., and C.B.; Writing - Original Draft, M.G.T., N.S., J.F.B., and J.D.K.; Writing - Review and Editing, M.G.T., N.S., J.F.B., C.B., M.W., N.K., A.M., N.J.H., and J.D.K.; Resources and supervision, N.J.H., A.M., and J.D.K.

\section{Additional Information}

Supplementary information accompanies this paper at https://doi.org/10.1038/s41598-018-20016-w.

Competing Interests: J.D.K. has financial interests in Amyris, Lygos, Constructive Biology, Demetrix, and Napigen.

Publisher's note: Springer Nature remains neutral with regard to jurisdictional claims in published maps and institutional affiliations.

(c) (i) Open Access This article is licensed under a Creative Commons Attribution 4.0 International License, which permits use, sharing, adaptation, distribution and reproduction in any medium or format, as long as you give appropriate credit to the original author(s) and the source, provide a link to the Creative Commons license, and indicate if changes were made. The images or other third party material in this article are included in the article's Creative Commons license, unless indicated otherwise in a credit line to the material. If material is not included in the article's Creative Commons license and your intended use is not permitted by statutory regulation or exceeds the permitted use, you will need to obtain permission directly from the copyright holder. To view a copy of this license, visit http://creativecommons.org/licenses/by/4.0/.

(C) The Author(s) 2018 